Arbeitens und Denkens hat er Bahnbrechendes geleistet, unüberbietbar, zeitlos gültig und anschlussfähig. Was uns bleibt ist, auf diesem Weg, den er der Soziologie unbeirrt gebahnt hat, weiter zu gehen. Radikal, kritisch und unbequem, so vielleicht lautet das Testament von Pierre Bourdieu, und genau so hätte auch das Programm einer kritischen Gesellschaftstheorie auszusehen. Nähmen wir es an und führten es in seinem Geiste weiter, erwiesen wir unserer Disziplin wie den zeitgenössischen Gesellschaften den besten Dienst.

Hans-Peter Müller

\section{Kongress der Deutschen Gesellschaft für Soziologie vom 7. bis 11. Oktober 2002 an der Universität Leipzig}

\section{Deutsche Gesellschaft für Soziologie:}

Vorsitz Prof. Jutta Allmendinger, Ph.D., www.soziologie.de

Lokale Veranstalter: Prof. Dr. Georg Vobruba und Prof. Dr. Kurt Mühler, Institut für Soziologie, Universität Leipzig

Kongress-Koordination: Susan Ulbricht, Universität Leipzig, Institut für Soziologie, Burgstr. 21, 04109 Leipzig, E-Mail: ulbricht@,dgs2002.de, Tel.0341/97 35 648, Fax $0341 / 9735669$

Das Thema des Kongresses „Entstaatlichung und soziale Sicherheit" umfasst ein weites Diskussionsfeld. "Entstaatlichung" ist dabei in zwei Dimensionen zu sehen: Territoriale Entstaatlichung meint eine Bedeutungsverschiebung weg von der nationalstaatlichen Ebene, einerseits hin zur supra- und internationalen Ebene, andererseits zur regionalen Ebene. Funktionelle Entstaatlichung ist als Übertragung von Staatsaufgaben auf nichtstaatliche Institutionen zu verstehen. Der Begriff "Soziale Sicherheit" beinhaltet alle Formen von Sicherheit in der Gesellschaft und geht damit weit über ein engeres sozialpolitisches Verständnis hinaus. Ein Auszug aus dem Veranstaltungsprogramm zeigt die Vielfalt der aktuellen Bezüge des Themas.

So werden beispielsweise die Abendveranstaltungen zu folgenden Themen durchgefulhrt:

- „Hat die Menschheit gemeinsame Interessen? Zur Konstruktion globaler Kollektivgüter" (Organisation: Prof. Dr. Bettina Heintz und Prof. Dr. Hans-Georg Soeffner)

- „Das Ende des mäzenatischen Staates? Beobachtungen aus kulturpolitischer Perspektive" (Organisation: Prof. Dr. Karl-Siegbert Rehberg)

- „Globalisierung ohne Wohlfahrtsstaaten?" (Organisation: Prof. Dr. Georg Vobruba)

- „Fundamentalistische Netzwerke als Bedrohung der Zivilisation?" (Organisation: Prof. Dr. Monika Wohlrab-Sahr)

Den Eröffnungsvortrag während der feierlichen Eröffnungsveranstaltung im Gewandhaus zu Leipzig hält Prof. Dr. Claus Offe. Als ReferentInnen der Mittagsvorlesungen werden Dieter Grimm, Alois Hahn, Arlie Hochschild, FranzXaver Kaufmann, John Meyer, Loïc Wacquant und Helmut Wiesenthal erwartet.

Weiterhin wird es 15 Plenarveranstaltungen geben, außerdem finden die Sektionssitzungen, Ad-hoc-Gruppen-Sitzungen, Postersessions und Foren statt. Darüber hinaus sind zwei öffentliche Podiumsdiskussionen geplant, deren Themen lauten „Die Öffentlichkeit der Soziologie“ (Organisation: Vorstand der DGS) sowie "Entstaatlichung von Ernährungsrisiken? Öffentliche Podiumsdiskussion zur Soziologie des Rinderwahns" (Organisation: Kai Brauer).

Ausführliche Informationen zu den genannten und weiteren Veranstaltungen gibt es im Vorprogramm, das unter oben genannter Adresse angefordert werden kann. Auch auf der KongressHomepage unter www.dgs2002.de finden Sie Anmeldeformulare zum Ausdrucken, alle Informationen des Vorprogramms, den Call for Papers, Neuigkeiten sowie viele zusätzliche Informationen und Links rund um den Kongress und Leipzig. Bis zum 1.6.2002 ist die Anmeldung zur ermäßigten Teilnahmegebühr möglich.

$\mathrm{Bis}$ bald in Leipzig! 